\title{
Cannabinoid CB2 Receptor Functional Variation (Q63R) is Associated with COVID-19 Severity: from Human Study to Molecular Docking
}

\section{Mostafa Rastgar}

Golestan University of Medical Sciences

Saeed Samadizadeh

Golestan University of Medical Sciences

Mohammad Yasaghi

Golestan University of Medical Sciences

Abdolvahab Moradi

Golestan University of Medical Sciences

Alijan Tabarraei

Golestan University of Medical Sciences

Vahid Salimi

Tehran University of Medical Sciences

Alireza Tahamtan ( $\nabla$ alireza.tmn@gmail.com )

Golestan University of Medical Sciences

\section{Research Article}

Keywords: SARS-CoV-2, COVID-19, CB2; Q63R, CNR2, docking

Posted Date: January 25th, 2021

DOI: https://doi.org/10.21203/rs.3.rs-144850/v1

License: (9) This work is licensed under a Creative Commons Attribution 4.0 International License. Read

Full License 


\section{Abstract}

Background: Evidence supports the role of host genetic diversity for the clinical course variation of coronavirus disease 2019 (COVID-19). Variation in the cannabinoid CB2 receptor gene (CNR2) could affect the endocannabinoids regulatory actions on the immune system, resulting in an increased risk of various inflammatory diseases. The present study investigated the relationship between the CNR2 rs35761398 (Q63R) functional variation and COVID-19 severity.

Results: A total of 200 Iranian COVID-19 patients (100 expired and 100 discharged) were enrolled in the study and genotyped through TaqMan assay. The co-dominant, dominant, recessive, over-dominant, and additive inheritance models were analyzed using SNPStats software. In silico molecular docking was also performed to simulate the effects of Q63R variation on CB2 binding with a ligand and with G-protein. A significant difference in the Q63R allele and genotype distributions was found between COVID-19 expired and discharged patients in co-dominant (OR: 3.33, 95\% Cl: 1.25-8.88, $p=0.043$ ), recessive (OR: 2.92, 95\% Cl: 1.16-7.33, $p=$ 0.017), and additive inheritance (OR: $1.62,95 \% \mathrm{Cl}: 1.06-2.48, p=0.025)$ models. The molecular docking results showed that the predicted structure of mutant CB2 (63R type) could not bind to G-protein in the correct position.

Conclusions: The data implied the involvement of the CNR2 gene in the severity of COVID-19 in Iranian patients. Identification of genes related to susceptibility and severity of COVID-19 may lead to specific targets for repurposing or drug development.

\section{Background}

Severe acute respiratory syndrome coronavirus 2 (SARS-CoV-2) as a newly emerging virus causes mild-tosevere respiratory disease, which has been named coronavirus disease 2019 (COVID-19) (1, 2). All people are susceptible to the virus infection, but there is considerable variation in the disease course and outcome among infected individuals (3). While many infected cases do not exhibit any symptoms, others proceed to develop COVID-19; however, severe illness and death occur only in a small minority of patients (4). Although our understanding of the SARS-CoV-2 and COVID-19 is still in its infancy, there is now strong evidence supporting the role of host genetic diversity alongside with other host, viral and environmental factors for the clinical course variation (5-12). Host genetic diversity could dictate the clinical response to respiratory viruses through susceptibility to viral infection, and propensity to develop harmful pulmonary inflammation (13). Finding a relationship between host genetics and the clinical outcome of SARS-CoV-2 infection may be necessary for identifying high-risk individuals.

The endocannabinoid (EC) system is a biological system composed of endogenous cannabinoids and their respective receptors, CB1 and CB2 (14). The system has been identified as a critical endogenous regulator of immune system homeostasis due to its effects on immune cells development, migration, proliferation, and effector functions (15). Cannabinoids have been proposed as a promising immunomodulator to reduce the SARS-CoV-2 immunopathology (16). Variations in the cannabinoid CB2 receptor gene (CNR2) could affect intracellular signaling and reduce the ECs function, which has been associated with an unbalanced immune response and increased risk of various inflammatory diseases (17-23). The CB2-Q63R polymorphism is a 
missense mutation of the second and third bases at codon 63 of the CNR2 gene, which leads to a Q/R substitution, causing a different polarization state of the protein (24). This variation has been shown to affect the response of CB2 to cannabinoids and differently modulate the EC-induced inhibition of lymphocyte proliferation (25). While evidence indicated that the mutation does not affect receptor-ligand binding (24), the exact mechanism behind this action is still unknown.

Focusing on the immunopathogenesis of SARS-CoV-2 and EC effects on the immune system, here we describe how variability in the CNR2 gene can conceivably explain variability in COVID-19 clinical phenotype. Besides, in silico molecular docking was performed to simulate the effects of CB2-Q63R variation on receptorligand and receptor-G-protein interaction. The data implied the involvement of the CNR2 gene in the severity of COVID-19 in Iranian patients.

\section{Results}

\section{Human study}

All patients were COVID-19 confirmed cases and hospitalized in the central hospital for COVID-19 patients in Gorgan city (Sayyad Medical and Educational Center). The details of the demographic, gender, age, and clinical data of all cases are presented in Table 1. The age distribution between expired patients (mean, 62.08 years) was significant compared with discharged patients (mean, 54.45 years) $(p<0.05)$. Moreover, the age distribution between female (mean, 63.12 years) and male (mean, 61.04 years) expired patients was significant compared with the discharged subjects (female mean, 55.64 years; male mean, 53.26 years) ( $p<$ 0.05). Of all patients enrolled in the study, the most observed symptoms were dyspnea (66.5\%), cough (66\%), fever or chill (59.9\%), sore throat (20.5\%), myalgia (16.5\%), ageusia and anosmia (14.5\%), nausea or vomiting $(10.5 \%)$, diarrhea $(7 \%)$, headache $(6 \%)$, chest pain $(5 \%)$, and fatigue $(3.5 \%)$. 
Table 1

Demographic, gender, age, and clinical data of subjects $(n=200)$

\begin{tabular}{|llll|}
\hline & All patients & Expired & Discharged \\
\hline Sex & & & \\
\hline Male, n (\%) & $100(50)$ & $50(25)$ & $50(25)$ \\
\hline Female, n (\%) & $100(50)$ & $50(25)$ & $50(25)$ \\
\hline Sex ratio & $1: 1$ & $1: 1$ & $1: 1$ \\
\hline Age (year) & & & \\
\hline Median & 59 & 54 & 63 \\
\hline Mean & 58.27 & 54.45 & 62.08 \\
\hline Clinical features & & & \\
\hline Cough & $132(66)$ & $66(66)$ & $66(66)$ \\
\hline Fever or chills & $119(59.5)$ & $62(62)$ & $57(57)$ \\
\hline Dyspnea & $133(66.5)$ & $74(74)$ & $59(59)$ \\
\hline Fatigue & $7(7)$ & $5(5)$ & $2(2)$ \\
\hline Myalgia & $10(5)$ & $8(8)$ & $2(2)$ \\
\hline Headache & $12(6)$ & $6(6)$ & $6(6)$ \\
\hline Ageusia and anosmia & $29(14.5)$ & $17(17)$ & $12(12)$ \\
\hline Sore throat & $41(20.5)$ & $20(20)$ & $21(21)$ \\
\hline Nausea or vomiting & $21(10.5)$ & $8(8)$ & $13(13)$ \\
\hline Diarrhea & & $7(7)$ & $7(7)$ \\
\hline Chest pain & & & \\
\hline
\end{tabular}

The allelic frequencies and the genotype distributions in expired and discharged patients are shown in Table 2. The frequencies of polymorphisms were found in the Hardy-Weinberg equilibrium among all subjects, expired and discharged patients $(p>0.05)$. When logistic regression was used to carry out association analysis after modeling the SNP effects, the SNP showed a significant difference in co-dominant (OR: 3.33, 95\% Cl: 1.25-8.88, $p=0.043$ ), recessive (OR: 2.92, 95\% Cl: 1.16-7.33, $p=0.017$ ), and additive models (OR: $1.62,95 \% \mathrm{Cl}: 1.06-2.48, p=0.025$ ) (Table 3). The recessive model was accepted as the best inheritance model to fit the data because of the smaller Akaike information criterion (AIC) value (275.6). A more significant difference was observed after adjusting for age (Table 3). No association between CB2-Q63R variants and demographic and clinical features was found (Table 4). It is important to note that the power of the test was 0.90486 . 
Table 2

Allele and genotype frequencies of Q63R polymorphism in subjects $(n=200)$

\begin{tabular}{|c|c|c|c|c|c|c|}
\hline & \multicolumn{2}{|c|}{ All patients } & \multicolumn{2}{|c|}{ Expired } & \multicolumn{2}{|c|}{ Discharged } \\
\hline Allele & Count & Proportion & Count & Proportion & Count & Proportion \\
\hline $\mathrm{R}$ & 253 & 0.63 & 137 & 0.68 & 116 & 0.58 \\
\hline Q & 147 & 0.37 & 63 & 0.32 & 84 & 0.42 \\
\hline \multicolumn{7}{|c|}{ Genotype } \\
\hline RR & 78 & 0.39 & 44 & 0.44 & 34 & 0.34 \\
\hline QR & 97 & 0.48 & 49 & 0.49 & 48 & 0.48 \\
\hline QQ & 25 & 0.12 & 7 & 0.07 & 18 & 0.18 \\
\hline
\end{tabular}


Table 3

Association of Q63R polymorphism with COVID-19 severity $(n=200$, crude analysis) under different inheritance models

\begin{tabular}{|c|c|c|c|c|c|c|c|c|c|}
\hline \multirow[t]{2}{*}{ Model } & \multirow[t]{2}{*}{ Genotype } & \multirow[t]{2}{*}{$\begin{array}{l}\text { Expired } \\
\text { (\%) }\end{array}$} & \multirow[t]{2}{*}{$\begin{array}{l}\text { Discharged } \\
\text { (\%) }\end{array}$} & $\begin{array}{l}\text { OR } \\
\text { (95\% } \\
\text { Cl) }\end{array}$ & $\begin{array}{l}P \\
\text { value }\end{array}$ & AIC & $\begin{array}{l}\text { OR } \\
\text { (95\% } \\
\text { Cl) }\end{array}$ & $\begin{array}{l}P \\
\text { value }\end{array}$ & AIC \\
\hline & & & & \multicolumn{3}{|c|}{ Not adjusted } & \multicolumn{3}{|c|}{ Adjusted by Age } \\
\hline \multirow{3}{*}{$\begin{array}{l}\text { Co- } \\
\text { dominant }\end{array}$} & $\mathrm{RR}$ & $44(44)$ & 34 (34) & 1.00 & \multirow[t]{3}{*}{0.043} & \multirow[t]{3}{*}{277} & 1.00 & \multirow[t]{3}{*}{0.015} & \multirow[t]{3}{*}{265} \\
\hline & QR & $49(49)$ & $48(49)$ & $\begin{array}{l}1.27 \\
(0.70- \\
2.31)\end{array}$ & & & $\begin{array}{l}1.44 \\
(0.77- \\
2.69)\end{array}$ & & \\
\hline & QQ & $7(7)$ & $18(18)$ & $\begin{array}{l}3.33 \\
(1.25- \\
8.88)\end{array}$ & & & $\begin{array}{l}4.25 \\
(1.52- \\
11.93)\end{array}$ & & \\
\hline \multirow[t]{2}{*}{ Dominant } & $\mathrm{RR}$ & $44(44)$ & 34 (34) & 1.00 & \multirow[t]{2}{*}{0.15} & \multirow[t]{2}{*}{279.2} & 1.00 & \multirow[t]{2}{*}{0.063} & \multirow[t]{2}{*}{267.9} \\
\hline & QR-QQ & $56(56)$ & $66(6)$ & $\begin{array}{l}1.53 \\
(0.86- \\
2.70)\end{array}$ & & & $\begin{array}{l}1.76 \\
(0.96- \\
3.20)\end{array}$ & & \\
\hline \multirow[t]{2}{*}{ Recessive } & RR-QR & $93(93)$ & $82(82)$ & 1.00 & \multirow[t]{2}{*}{0.017} & \multirow[t]{2}{*}{275.6} & 1.00 & \multirow[t]{2}{*}{0.0079} & \multirow[t]{2}{*}{264.3} \\
\hline & QQ & 7 (7) & $18(18)$ & $\begin{array}{l}2.92 \\
(1.16- \\
7.33)\end{array}$ & & & $\begin{array}{l}3.44 \\
(1.32- \\
8.99)\end{array}$ & & \\
\hline \multirow{2}{*}{$\begin{array}{l}\text { Over- } \\
\text { dominant }\end{array}$} & RR-QQ & $51(52)$ & $52(52)$ & 1.00 & \multirow[t]{2}{*}{0.89} & \multirow[t]{2}{*}{281.2} & 1.00 & \multirow[t]{2}{*}{0.94} & \multirow[t]{2}{*}{271.4} \\
\hline & QR & $49(49)$ & $48(48)$ & $\begin{array}{l}0.96 \\
(0.55- \\
1.67)\end{array}$ & & & $\begin{array}{l}1.02 \\
(0.58- \\
1.81)\end{array}$ & & \\
\hline $\begin{array}{l}\text { Log- } \\
\text { additive }\end{array}$ & - & - & - & $\begin{array}{l}1.62 \\
(1.06- \\
2.48)\end{array}$ & 0.025 & 276.3 & $\begin{array}{l}1.83 \\
(1.16- \\
2.88)\end{array}$ & 0.0073 & 264.2 \\
\hline
\end{tabular}


Table 4

Demographic, gender, and clinical form according to Q63R polymorphism

\begin{tabular}{|c|c|c|c|c|c|}
\hline & $\mathrm{RR}$ & QR & $\mathrm{QQ}$ & Total & $P$-value \\
\hline \multicolumn{6}{|l|}{ Gender } \\
\hline Male & $44(56.4)$ & $43(44.3)$ & $13(48)$ & $100(50)$ & \multirow[t]{3}{*}{0.277} \\
\hline Female & $34(43.6)$ & $54(55.7)$ & $12(52)$ & $100(50)$ & \\
\hline Total & $78(100)$ & $97(100)$ & $25(100)$ & $200(100)$ & \\
\hline \multicolumn{6}{|l|}{ Symptoms } \\
\hline Cough & $55(70.5)$ & $60(61.9)$ & $17(68)$ & $132(66)$ & 0.474 \\
\hline Fever or chills & $45(57.7)$ & $57(58.8)$ & $17(68)$ & $119(59.9)$ & 0.645 \\
\hline Dyspnea & $57(73.1)$ & $61(62.9)$ & $15(60)$ & $133(66.5)$ & 0.278 \\
\hline Fatigue & $5(6.4)$ & $2(2.1)$ & $0(0)$ & $7(3.5)$ & 0.178 \\
\hline Myalgia & $13(16.7)$ & $17(17.5)$ & $3(12)$ & $33(16.5)$ & 0.801 \\
\hline Headache & $5(6.4)$ & $7(7.2)$ & $0(0)$ & $12(6)$ & 0.392 \\
\hline Ageusia and anosmia & $8(10.3)$ & 19 (19.6) & $2(8)$ & $29(14.5)$ & 0.135 \\
\hline Sore throat & $15(10.2)$ & $22(22.7)$ & $4(16)$ & $41(20.5)$ & 0.715 \\
\hline Nausea or vomiting & $10(12.8)$ & $11(11.3)$ & $0(0)$ & $21(10.5)$ & 0.178 \\
\hline Diarrhea & $6(7.7)$ & $6(6.2)$ & $2(8)$ & $14(7)$ & 0.907 \\
\hline Chest pain & $2(2.6)$ & $6(6.2)$ & $2(8)$ & $10(5)$ & 0.420 \\
\hline
\end{tabular}

\section{Molecular docking}

The I-TASSER server predicted five models for the submitted sequences, and we selected the best predicted 3D structure of mutant CB2 (63R) with c-score, i.e., -0.9 with a 0.74 TM-score. C-score shows the quality of the predicted model by I-TASSER, and a model with a higher c-score signifies a higher confidence predicted model. TM-score shows the similarity between the predicted model and the template (6PT0), and the TM-score of more than 0.5 exhibits the correct topology of the predicted model. The best model of homology with $100 \%$ confidence and $94 \%$ identity were selected. The 3D structure of the predicted $63 \mathrm{R}$ models and wild type CB2 (PDB ID 6PT0, Chain R) are shown in Figure 1. Ramachandran plots are shown in Figure 2.

Molecular docking was performed to examine the binding affinity and possible binding pocket of 2-AG with both the built CB2-63R models and wild-type CB2-Q63. The binding energy between 2-AG and CB2-Q63, 2-AG and I-TASSER built model, and 2-AG and Phyre2 built model were -7.5, -6.9, and $-7.0 \mathrm{Kcal} / \mathrm{mol}$, respectively. Lower binding energy indicates better interaction, more affinity, and more stability between ligand and protein. 
The binding position, hydrogen bonds, alkyl bonds, van der Waals forces, and binding residues are shown in Figure 3.

The HDOCK server was used for protein-protein docking and the possibility of interaction between CB2 and Gprotein. The docking results for wild-type CB2 and G-protein were -452.97 docking score and $1.06 \mathrm{~A}^{\circ}$ rootmean-square deviation (RMSD), and the binding affinity was $-13.3(\mathrm{Kcal} / \mathrm{mol})$. These data showed the correct binding position of G-protein and CB2-Q63 after docking compared with CB2 structure coupled with G-protein (PDB ID: 6PT0). The docking results for the I-TASSER model and G-protein were -350.14 docking score and $80.32 \mathrm{~A}^{\circ}$ ligand RMSD, and for the Phyre2 model and G-protein were -322.83 docking score and $192.66 \mathrm{~A}^{\circ}$ ligand RMSD. These results show that both predicted structures of mutant CB2 cannot bind to G-protein in the correct position. Interaction between both CB2-Q63 and CB2-63R models and G-protein is shown in Figure 4.

\section{Discussion}

As a newly emerging virus, all people are susceptible to SARS-CoV-2 infection, though the nature and severity of COVID-19 vary significantly among cases. Notably, reported disease burden and case fatality rates differ considerably from one country to another (36). The exact influence of host genetic makeup in this variation has remained mostly unknown. The importance of host genetics' contribution in differential responses to SARS-CoV-2 is highlighted by a modeling study, revealing that $50 \%$ of the variance of the 'predicted COVID-19' phenotype is due to genetic factors (6). For example, studies reported that the variable expression pattern and genetic variation in angiotensin-converting enzyme 2 (ACE2), as a functional receptor for SARS-CoV-2, might be associated with the susceptibility to infection and severity of the disease (37). Notably, a recent genomewide association study revealed that critical illness in COVID-19 is related to host antiviral defense mechanisms (IFNAR2 and OAS genes) and mediators of inflammatory organ damage (DPP9, TYK2, and CCR2) (38). Thus, genes related to immune responses are of particular interest for our understanding of predisposition to severe COVID-19 because of the immunopathology of SARS-CoV-2.

The EC system has received much attention due to its regulatory roles in immune response and its effects on immune-associated disease progression (39). There is evidence supporting the system's specific involvement in respiratory viral associated immunopathology and in modulating inflammation following infection. We previously provided evidence that the EC system plays an essential role during respiratory syncytial virus (RSV) infection in humans and mice (17). Other studies also showed that the lack of cannabinoid receptors could increase inflammation and tissue damage following influenza virus infection, and their activation can impair immune responses induced by the virus (40-42). Such data supported the idea of using cannabinoids as a potential therapeutic approach in COVID-19 patients $(16,43)$. The rationale behind the current study was based on SARS-CoV-2 immunopathology, together with the data available on the immune regulatory role of CB2 signaling.

The CB2-Q63R polymorphism is caused by two missense mutations in the CNR2 gene changing CAA to CGG, which lead to a substitution of an uncharged polar amino acid (glutamine) with a positively charged polar amino acid (arginine) at position 63 located at the first intracellular loop of the CB2 receptor (24). Studies indicated that the CB2-63R variant has less functions than $63 \mathrm{Q}$ in the modulation of immune responses, especially T-cell proliferation (25). While the exact mechanism is unknown, a study reported that the signal 
intensity caused by $63 \mathrm{R}$ activation is relatively weaker than that caused by $63 \mathrm{Q}$ activation (44). The present study reports for the first time, the association between the CB2 receptor and COVID-19 severity. A significant difference in the Q63R allele and genotype distributions was found between COVID-19 expired and discharged patients (Table 2). The co-dominant, recessive, and additive inheritance models showed a significant association between Q63R and COVID-19 severity (Table 3). According to the co-dominant model, RR subjects showed a risk for developing severe COVID-19 more than thrice of QQ subjects.

The association between the CB2-Q63R variation and autoimmune conditions such as thrombocytopenic purpura (45), celiac disease (23), juvenile idiopathic arthritis (19), inflammatory bowel disease (46), and rheumatoid arthritis (22) has been reported. The data reported by the current authors have been implied the involvement of the Q63R variation in susceptibility to multiple sclerosis in Iranian patients (18). Interestingly, our previous study showed that the inflammatory response to virus is more inhibited in cases with QQ variants, allowing the virus to replicate and induce severe infection (17). In the case of SARS-CoV-2 infection, while a robust innate immune response is essential to eliminate viral pathogens, a prolonged or dysregulated/exuberant manner can damage the respiratory tract (47). The current results are consistent with previous studies that showed a reduced EC-induced modulation of the immune system in human subjects carrying the RR variant of CB2 compared with those having the QQ variant $(2,19,23,45,46)$.

Importantly, experimental data from previous competition ligand binding assay showed that the binding affinity of 2-AG and CB2-63R is similar to CB2-Q63, but CB2-63R had a significantly lower maximum response after binding to 2-AG compared with Q63 type (24). Our molecular docking results confirm that the CB2-63R induces a more inadequate response to ligand binding. The biological effects of cannabinoids are mediated through the activation of G-protein-coupled cannabinoid receptors (48). G-proteins act as adaptors that link Gprotein-coupled receptors (GPCRs) to other signaling and regulatory proteins to operate or modulate intracellular signaling pathways (15). The molecular docking results showed that the predicted structures of mutant CB2 could not bind to G-protein in the correct position, resulting in EC signaling dysfunction. This data is consistent with Wang et al., finding that CB2-63R induces lower signaling transduction than CB2-63Q in human primary T-cells (44). The CB2 receptor is predominantly expressed in the immune and immune-derived cells, and its activation indirectly affects viral infections by altering host immune responses, particularly inflammation, along different signaling pathways $(49,50)$.

\section{Conclusion}

Data from the current study points toward host genetic involvement in the severity of COVID-19. Host genetic certainly affects the balance of immune responses during any viral immunopathogenesis, leading to different clinical phenotypes. Results indicate that people with CB2-63R variant are more prone to develop severe COVID-19. Considering the potential of this polymorphism as a biomarker in COVID-19 severity, there is an urgent need to deepen these findings through further studies. Regarding the sample size limitation and different genetic backgrounds in various populations, other studies using whole-genome sequencing with a large cohort for multiple people would be required. Identification of genes related to susceptibility and severity of COVID-19 may lead to specific targets for repurposing or drug development. We hope that, with great efforts, scientific support, and information sharing, the overcoming of COVID-19 will come soon. 


\section{Methods}

\section{Human study}

A total of 200 Iranian COVID-19 patients were included in this study. The case group consisted of 100 expired cases (50 women and 50 men) with a mean age of 62.08 years, and the control group consisted of 100 discharged cases ( 50 women and 50 men) with a mean age of 54.45 years. All COVID-19 patients were confirmed by real-time RT-PCR assay targeting the SARS-CoV-2 nucleoprotein (N) and ORF1ab genes (Pishtazteb, Iran). A clinical questionnaire was developed for this study and used to collect data from all patients, including gender, age, and clinical symptoms at admission (Additional file 1). Nasopharyngeal samples were collected from all patients and divided into two groups according to their disease outcome. All of the subjects in this study were from Golestan, Province, and had the same geographical origin, and none were related.

Genomic DNA was extracted from the collected nasopharyngeal samples using a DNA extraction kit following manufacturers' instructions (GeneAll, South Korea). Extracted samples were genotyped for the CNR2 rs35761398 (Q63R) variant using a TaqMan assay with commercial primers/probes (Thermo Fisher, USA). The reaction conditions were as follows: $95^{\circ} \mathrm{C}$ for 4 min, followed by 50 cycles of $95^{\circ} \mathrm{C}$ for $15 \mathrm{~s}$, and $60{ }^{\circ} \mathrm{C}$ for 90 s. Both PCR and post-PCR allelic discrimination was performed on an ABI PRISM 7300 system (Applied Biosystems, USA). Genotypes of ten percent random samples per each group were confirmed by direct PCR sequencing as described previously (18).

The demographic and clinical data were analyzed with SPSS23 software (IBM, Chicago, IL). The HardyWeinberg equilibrium (HWE) and differences in allele and genotypic frequencies were calculated using the SNPStats software (a web tool for the analysis of association studies:

http://bioinfo.iconcologia.net/SNPstats) (26). Inheritance models such as co-dominant, dominant, recessive, over-dominant, and log-additive were analyzed using the SNPStats software. The OR was adjusted by age in the logistic regression model. The power of the test was calculated using G-power 3.1.9.4 software (Universitat Kiel, Germany). Odds ratios (OR) and 95\% confidence intervals (Cl) were calculated, and $p$-values less than 0.05 were considered statistically significant.

\section{Molecular docking}

To predict the 3-dimensional (3D) structure of mutant CB2 (63R), CB2 protein sequence (NCBI Reference Sequence: NP_001832.1) with a change in position 63 was submitted to I-TASSER server (https://zhanglab.ccmb.med.umich.edu/I-TASSER), which use a hierarchical approach to protein structure prediction (27). The best model is selected from the output based on the confidence score (c-score). The Phyre2 server was also used to predict the 3D structure of 63R based on homology (http://www.sbg.bio.ic.ac.uk/phyre2/html/page.cgi?id=index) (28). The best model based on confidence and identity was selected as a homology model. Both models were submitted to ModRefiner (https://zhanglab.ccmb.med.umich.edu/ModRefiner) for atomic-level, high-resolution protein structure refinement to energy minimization and structure refinement (29). Ramachandran validation was performed 
using the MolProbity server (http://molprobity.biochem.duke.edu/) for backbone and structure validation of the built 3D models (30).

The 3D coordinates of wild-type CB2 (PDB code: 6PT0) were downloaded from Protein Data Bank (https://www.rcsb.org) in PDB format that opened in UCSF Chimera software (31), and R chain (CB2) selected and prepared for docking (removed water, added polar hydrogens and added charge). The same preparation is used for I-TASSER and Phyre2 built models of mutant CB2. The 2-Arachidonoylglycerol (2-AG) was selected as the main endogenous agonist of CB2. The SDF file was downloaded from the PubChem database (https://pubchem.ncbi.nlm.nih.gov/). The 2D SDF file was converted to a 3D PDB file using OpenBabel 2.4.0 (32) and opened in UCSF Chimera.

Following the preparation of receptors and ligand, blind docking (as the binding pocket of CB2 is unknown) were performed using Autodock Vina version 1.1.2 (33). Dock results were analyzed with UCSF Chimera, and the top score poses were selected, and the binding pocket was visualized in Discovery Studio. Protein-protein docking was performed to investigate the interaction between receptors and G-protein using HDOCK server (http://hdock.phys.hust.edu.cn) (34), which is based on a hybrid algorithm of template-based modeling and ab initio free docking. At first, HDOCK was performed for wild-type CB2 and G-protein, then the same protocol was carried out for mutant models and $G$ protein. Best poses with best docking score selected and visualized in UCSF Chimera and the binding energy were predicted using PRODIGY server (https://wenmr.science.uu.nl/prodigy) (35).

\section{Abbreviations}

Severe acute respiratory syndrome coronavirus 2, SARS-CoV-2; coronavirus disease 2019, COVID-19; endocannabinoid, EC; cannabinoid receptor 2, CB2; cannabinoid CB2 receptor gene, CNR2; reverse transcription-polymerase chain reaction, RT-PCR; Hardy-Weinberg equilibrium, HWE; 3-dimensional, 3D; protein data bank, PDB; odds ratios, OR; confidence intervals, Cl; Akaike information criterion, AIC; root-mean-square deviation, RMSD; angiotensin-converting enzyme 2, ACE2; during respiratory syncytial virus, RSV; G-proteincoupled receptors, GPCRs;

\section{Declarations}

\section{Ethics approval and consent to participate}

This study was approved by the ethic committee of the Golestan University of Medical Sciences (IR.GOUMS.REC.1399.005). All methods were performed in accordance with the relevant guidelines and regulations. Informed written consent was obtained from all subjects or, if subjects are under 18 , from a parent and/or legal guardian.

\section{Consent for publication}

Not applicable. 


\section{Availability of data and materials}

The datasets during and/or analyzed during the current study are available from the corresponding author on reasonable request.

\section{Competing interests}

The authors declare that they have no competing interests.

\section{Funding}

No specific funding was obtained for this work.

\section{Authors' contributions}

A.T conceptualized and designed the study. M.R, S.S and M.Y collected samples and did the experiment. A.T and M.R analyzed the collected data. MR drafted the manuscript. A.T, A.M, A.Tb, and V.S evaluated and edited the manuscript. All authors have read and approved the final manuscript.

\section{Acknowledgements}

The authors would like to thank Golestan University of Medical Sciences and SARS-CoV-2 laboratory and nursing team that are fighting against the illness.

\section{References}

1. Wang C, Horby PW, Hayden FG, Gao GF. A novel coronavirus outbreak of global health concern. Lancet. 2020;395(10223),470-3.

2. Chen N, Zhou M, Dong X, Qu J, Gong F, Han Y, et al. Epidemiological and clinical characteristics of 99 cases of 2019 novel coronavirus pneumonia in Wuhan, China, a descriptive study. Lancet. 2020;395(10223),507-13.

3. Teymoori-Rad M, Samadizadeh S, Tabarraei A, Moradi A, Shahbaz MB, Tahamtan A. Ten challenging questions about SARS-CoV-2 and COVID-19. Expert Rev Respir Med. 2020.

4. Grasselli G, Zangrillo A, Zanella A, Antonelli M, Cabrini L, Castelli A, et al. Baseline characteristics and outcomes of 1591 patients infected with SARS-CoV-2 admitted to ICUs of the Lombardy Region, Italy. Jama. 2020;323(16),1574-81.

5. Sharif-Askari NS, Sharif-Askari FS, Alabed M, Temsah M-H, Al Heialy S, Hamid Q, et al. Airways Expression of SARS-CoV-2 Receptor, ACE2, and TMPRSS2 Is Lower in Children Than Adults and Increases with Smoking and COPD. Mol Ther Methods Clin Dev. 2020;18,1-6. 
6. Williams FM, Freydin M, Mangino M, Couvreur S, Visconti A, Bowyer RC, et al. Self-reported symptoms of covid-19 including symptoms most predictive of SARS-CoV-2 infection, are heritable. medRxiv. 2020.

7. Zhao J, Yang Y, Huang H-P, Li D, Gu D-F, Lu X-F, et al. Relationship between the ABO Blood Group and the COVID-19 Susceptibility. medRxiv. 2020.

8. Korber B, Fischer WM, Gnanakaran S, Yoon H, Theiler J, Abfalterer W, et al. Tracking changes in SARSCoV-2 Spike: evidence that D614G increases infectivity of the COVID-19 virus. Cell. 2020;182(4),812-27. e19.

9. Liu Y, Yan L-M, Wan L, Xiang T-X, Le A, Liu J-M, et al. Viral dynamics in mild and severe cases of COVID19. Lancet Infect. Dis. 2020.

10. Tan J, Mu L, Huang J, Yu S, Chen B, Yin J. An initial investigation of the association between the SARS outbreak and weather: with the view of the environmental temperature and its variation. JECH 2005;59(3), 186-92.

11. Lin K, Fong DY-T, Zhu B, Karlberg J. Environmental factors on the SARS epidemic: air temperature, passage of time and multiplicative effect of hospital infection. Epidemiol Infect. 2006;134(2),223-30.

12. Roxby AC, Greninger AL, Hatfield KM, Lynch JB, Dellit TH, James A, et al. Detection of SARS-CoV-2 among residents and staff members of an independent and assisted living community for older adults-Seattle, Washington, 2020. MMWR 2020;69(14),416.

13. Tahamtan A, Samadizadeh S, Rastegar M, Nakstad B, Salimi V. Respiratory syncytial virus infection: why does disease severity vary among individuals? Expert Rev Respir Med. 2020;14(4),415-23.

14. Lu H-C, Mackie K. An introduction to the endogenous cannabinoid system. Biol Psychiatry. 2016;79(7),516-25.

15. Tahamtan A, Tavakoli-Yaraki M, Rygiel TP, Mokhtari-Azad T, Salimi V. Effects of cannabinoids and their receptors on viral infections. J Med Virol. 2016;88(1),1-12.

16. Tahamtan A, Tavakoli-Yaraki M, Salimi V. Opioids/cannabinoids as a potential therapeutic approach in COVID-19 patients. Expert Rev Respir Med. 2020;14(10),965-7.

17. Tahamtan A, Samieipoor Y, Nayeri FS, Rahbarimanesh AA, Izadi A, Rashidi-Nezhad A, et al. Effects of cannabinoid receptor type 2 in respiratory syncytial virus infection in human subjects and mice. Virulence. 2018;9(1),217-30.

18. Tahamtan A, Rezaiy S, Samadizadeh S, Moradi A, Tabarraei A, Javid N, et al. Cannabinoid CB2 Receptor Functional Variation (Q63R) Is Associated with Multiple Sclerosis in Iranian Subjects. J Mol Neurosci. 2020;70(1),26-31.

19. Bellini G, Olivieri AN, Grandone A, Alessio M, Gicchino M, Nobili B, et al. Association between cannabinoid receptor type 2 Q63R variant and oligo/polyarticular juvenile idiopathic arthritis. Scand J Rheumatol. 2015;44(4),284-7.

20. Gouda HM, Kamel NRM. Cannabinoid CB2 receptor gene (CNR2) polymorphism is associated with chronic childhood immune thrombocytopenia in Egypt. Blood Coagul. Fibrinolysis 2013;24(3),247-51.

21. Strisciuglio C, Bellini G, Miele E, Martinelli M, Cenni S, Tortora C, et al. Cannabinoid receptor 2 functional variant contributes to the risk for pediatric inflammatory bowel disease. J Clin Gastroenterol. 2018;52(5),e37-e43. 
22. Ismail M, Khawaja G. Study of cannabinoid receptor 2 Q63R gene polymorphism in Lebanese patients with rheumatoid arthritis. Clin Rheumatol. 2018;37(11),2933-8.

23. Rossi F, Bellini G, Tolone C, Luongo L, Mancusi S, Papparella A, et al. The cannabinoid receptor type 2 Q63R variant increases the risk of celiac disease: implication for a novel molecular biomarker and future therapeutic intervention. Pharmacol Res. 2012;66(1),88-94.

24. Carrasquer A, Nebane NM, Williams WM, Song Z-H. Functional consequences of nonsynonymous single nucleotide polymorphisms in the CB2 cannabinoid receptor. Pharmacogenet. Genomics 2010;20(3),15766.

25. Sipe JC, Arbour N, Gerber A, Beutler E. Reduced endocannabinoid immune modulation by a common cannabinoid 2 (CB2) receptor gene polymorphism: possible risk for autoimmune disorders. J Leukoc Biol. 2005;78(1),231-8.

26. Solé X, Guinó E, Valls J, Iniesta R, Moreno V. SNPStats: a web tool for the analysis of association studies. Bioinformatics. 2006;22(15),1928-9.

27. Roy A, Kucukural A, Zhang Y. I-TASSER: a unified platform for automated protein structure and function prediction. Nat Protoc. 2010;5(4),725-38.

28. Kelley LA, Mezulis S, Yates CM, Wass MN, Sternberg MJE. The Phyre2 web portal for protein modeling, prediction and analysis. Nat Protoc. 2015;10(6),845-58.

29. Xu D, Zhang Y. Improving the physical realism and structural accuracy of protein models by a two-step atomic-level energy minimization. Biophys J. 2011;101(10),2525-34.

30. Ramachandran GN, Ramakrishnan C, Sasisekharan V. Stereochemistry of polypeptide chain configurations. J Mol Biol. 1963;7(1),95-9.

31. Pettersen EF, Goddard TD, Huang CC, Couch GS, Greenblatt DM, Meng EC, et al. UCSF Chimera-a visualization system for exploratory research and analysis. J Comput Chem. 2004;25(13),1605-12.

32. O'Boyle NM, Banck M, James CA, Morley C, Vandermeersch T, Hutchison GR. Open Babel: An open chemical toolbox. J Cheminformatics. 2011;3(1),33.

33. Trott O, Olson AJ. AutoDock Vina: improving the speed and accuracy of docking with a new scoring function, efficient optimization, and multithreading. J Comput Chem. 2010;31(2),455-61.

34. Yan Y, Zhang D, Zhou P, Li B, Huang S-Y. HDOCK: a web server for protein-protein and protein-DNA/RNA docking based on a hybrid strategy. Nucleic Acids Res. 2017;45(W1),W365-W73.

35. Xue LC, Rodrigues JP, Kastritis PL, Bonvin AM, Vangone A. PRODIGY: a web server for predicting the binding affinity of protein-protein complexes. Bioinformatics. 2016;32(23),3676-8.

36. Karadag E. Increase in COVID-19 cases and case-fatality and case-recovery rates in Europe: A crosstemporal meta-analysis. J Med Virol. 2020;92(9),1511-7.

37. Devaux CA, Rolain JM, Raoult D. ACE2 receptor polymorphism: Susceptibility to SARS-CoV-2, hypertension, multi-organ failure, and COVID-19 disease outcome. J Microbiol Immunol Infect. 2020;53(3),425-35.

38. Pairo-Castineira E, Clohisey S, Klaric L, Bretherick AD, Rawlik K, Pasko D, Walker S, Parkinson N, Fourman M, Russell C, Furniss J, et al. Genetic mechanisms of critical illness in Covid-19. Nature. 2020. 
39. Klein TW, Newton C, Friedman H. Cannabinoid receptors and immunity. Immunol Today. 1998;19(8),37381.

40. Karmaus PWF, Chen W, Crawford R, Kaplan BLF, Kaminski NE. $\Delta$ 9-tetrahydrocannabinol impairs the inflammatory response to influenza infection: role of antigen-presenting cells and the cannabinoid receptors 1 and 2. Toxicol Sci. 2013;131(2),419-33.

41. Buchweitz JP, Karmaus PW, Williams KJ, Harkema JR, Kaminski NE. Targeted deletion of cannabinoid receptors $\mathrm{CB} 1$ and $\mathrm{CB} 2$ produced enhanced inflammatory responses to influenza A/PR/8/34 in the absence and presence of Delta9-tetrahydrocannabinol. J Leukoc Biol. 2008;83(3),785-96.

42. Karmaus PWF, Chen W, Crawford RB, Harkema JR, Kaplan BLF, Kaminski NE. Deletion of cannabinoid receptors 1 and 2 exacerbates APC function to increase inflammation and cellular immunity during influenza infection. J Leukoc Biol. 2011;90(5),983-95.

43. Rossi F, Tortora C, Argenziano M, Di Paola A, Punzo F. Cannabinoid Receptor Type 2: A Possible Target in SARS-CoV-2 (CoV-19) Infection? Int. J Mol Sci. 2020;21(11).

44. Wang J, Xu J, Liu J, Zhu H, Peng Y, Ding Z-M, et al. Genetic variant Q63R of cannabinoid receptor 2 causes differential ERK phosphorylation in human immune cells. Genet. Test Mol Biomarkers. 2018;22(5),320-6.

45. Rossi F, Mancusi S, Bellini G, Roberti D, Punzo F, Vetrella S, et al. CNR2 functional variant (Q63R) influences childhood immune thrombocytopenic purpura. Haematologica. 2011;96(12),1883-5.

46. Strisciuglio C, Bellini G, Miele E, Martinelli M, Cenni S, Tortora C, et al. Cannabinoid receptor 2 functional variant contributes to the risk of pediatric inflammatory bowel disease. Dig Liver Dis. 2016;48,e269.

47. Conti P, Ronconi G, Caraffa A, Gallenga CE, Ross R, Frydas I, et al. Induction of pro-inflammatory cytokines (IL-1 and IL-6) and lung inflammation by Coronavirus-19 (COVI-19 or SARS-CoV-2): anti-inflammatory strategies. J Biol Regul Homeost Agents. 2020;34(2),327-31.

48. Piomelli D. The molecular logic of endocannabinoid signalling. Nat Rev Neurosci. 2003;4(11),873-84.

49. Yao B, Mackie K. Endocannabinoid receptor pharmacology. Curr Top Behav Neurosci. 2009;1,37-63.

50. Rieder SA, Chauhan A, Singh U, Nagarkatti M, Nagarkatti P. Cannabinoid-induced apoptosis in immune cells as a pathway to immunosuppression. Immunobiology. 2010;215(8),598-605.

\section{Figures}




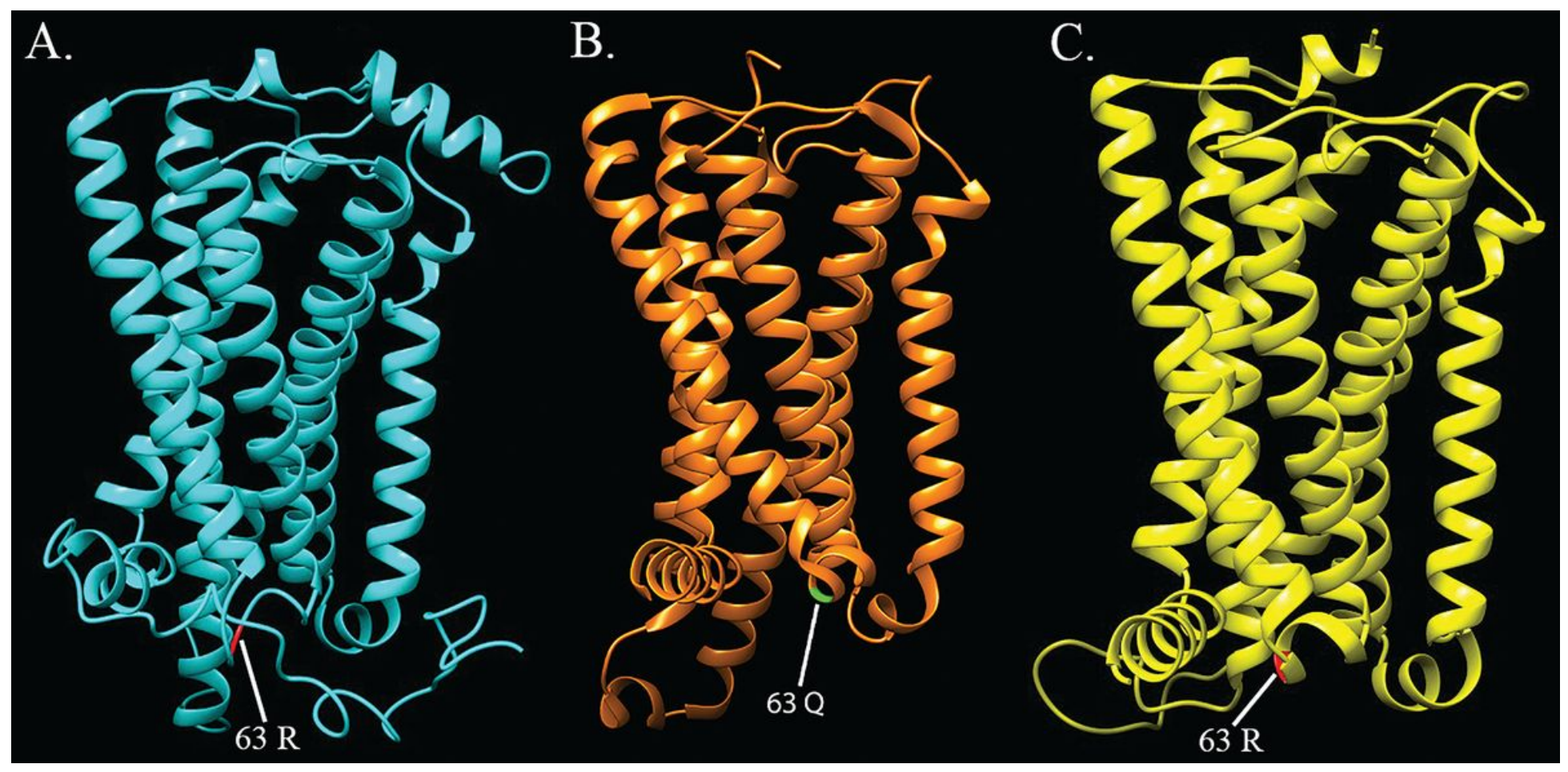

Figure 1

The 3-dimensional structure of A) the I-TASSER built model of mutant CB2 (63R), B) wild type CB2 (PDB ID: $6 \mathrm{PTO}, \mathrm{R}$ chain), and $\mathrm{C}$ ) the Phyre2 built model of 63R. The position of amino acid 63 is shown.

A.

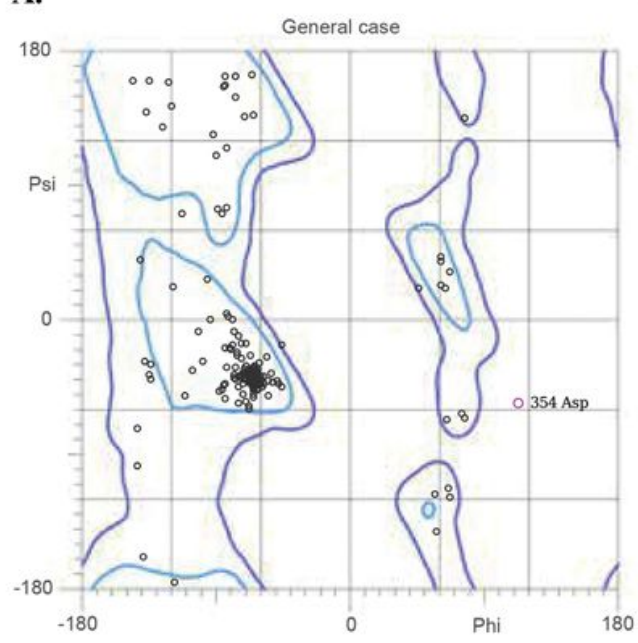

$92.7 \%(332 / 358)$ of all residues were in favored $(98 \%)$ regions. $98.9 \%(354 / 358)$ of all residues were in allowed $(>99.8 \%)$ regions.

There were 4 outliers (phi, psi):

176 Pro $(48.3,38.1)$

333 Pro $(-126.0,-122.5)$

$347 \operatorname{Thr}(102.8,-49.9)$

354 Asp $(113.3,-56.3)$
B.

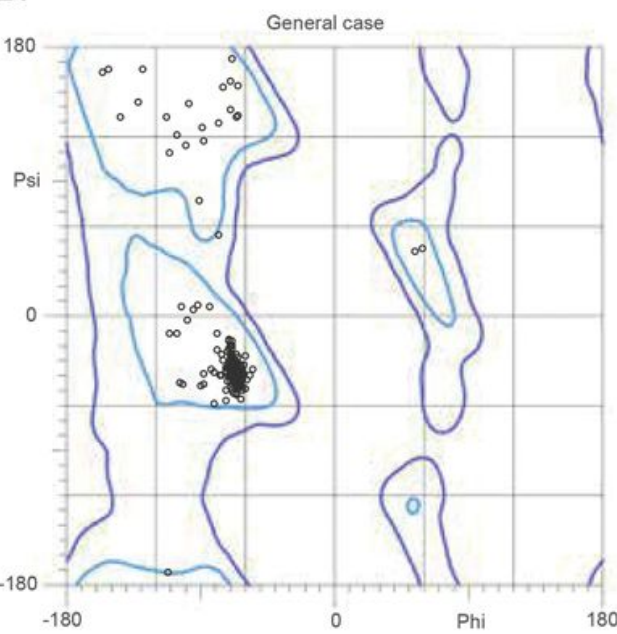

$\mathbf{9 8 . 6 \%}$ (292/296) of all residues were in favored $(98 \%)$ regions. $100.0 \%(296 / 296)$ of all residues were in allowed $(>99.8 \%)$ regions.

There were no outliers.
C.

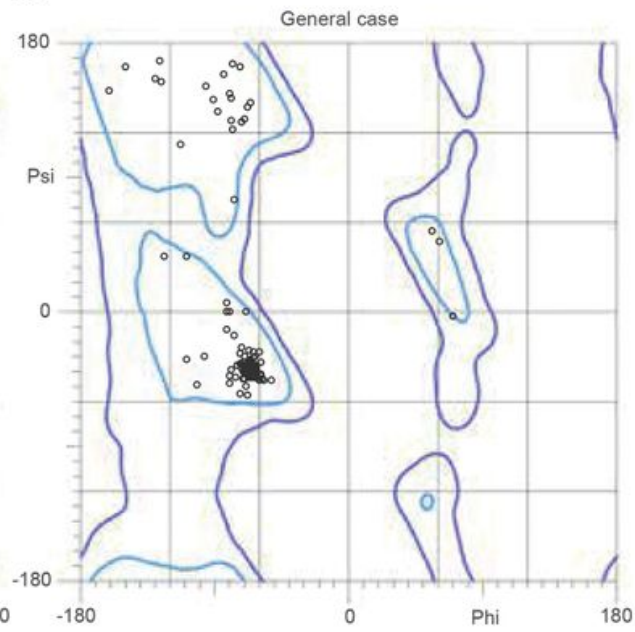

$99.3 \%(296 / 298)$ of all residues were in favored $(98 \%)$ regions. $100.0 \%(298 / 298)$ of all residues were in allowed $(>99.8 \%)$ regions.

There were no outliers.

\section{Figure 2}

Ramachandran plot general cases of $A$ ) the I-TASSER built model of mutant CB2 (63R), B) wild type CB2 (PDB ID: 6PT0, R chain), and C) the Phyre2 built model of 63R. Favored and allowed residues and the outliers are shown. 


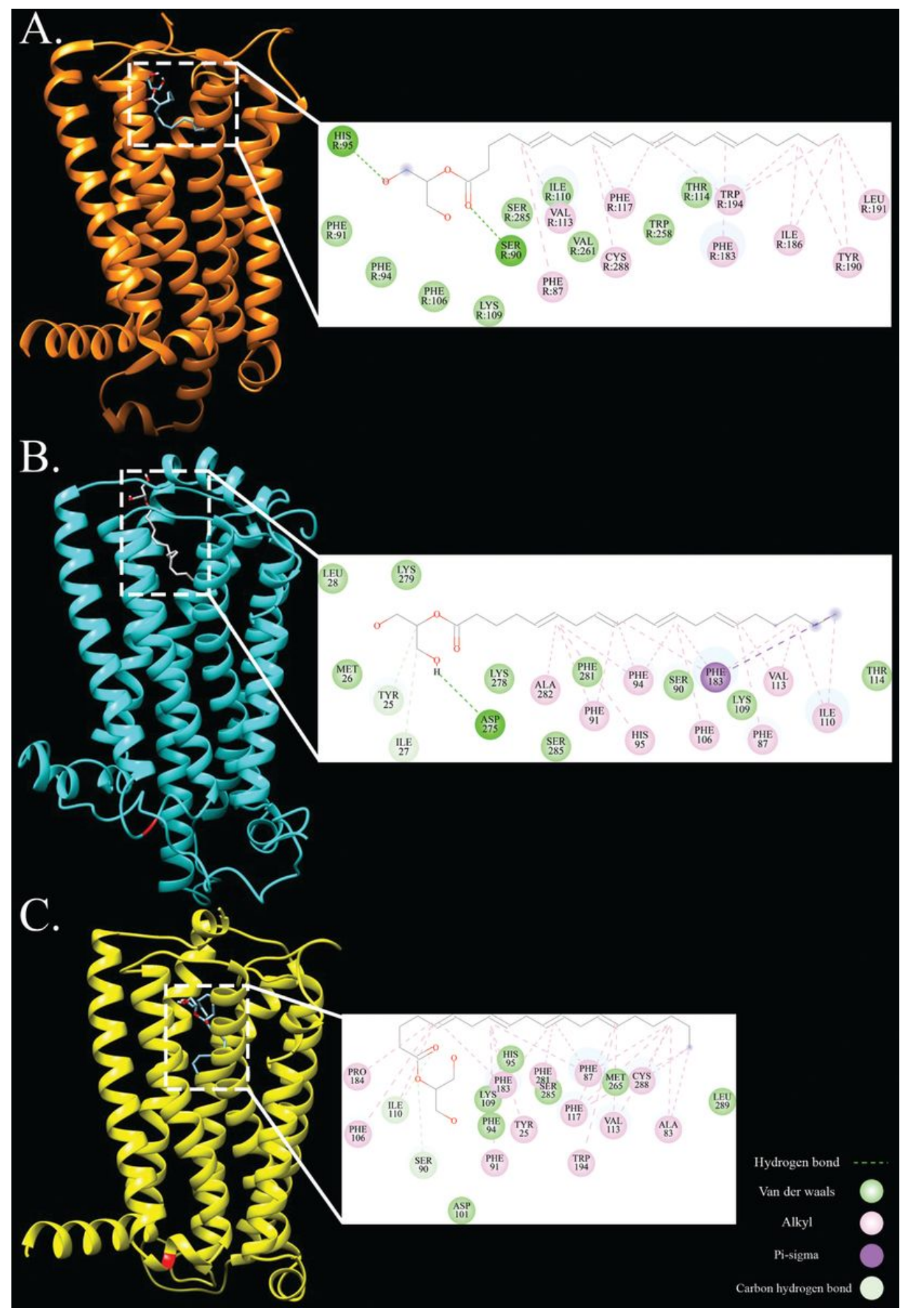

\section{Figure 3}

Visualization of docking analysis of 2-Arachidonoylglycerol (2-AG) with A) wild type CB2, B) I-TASSER built mutant CB2 model, and C) Phyre2 built mutant CB2 model. The binding position, hydrogen bonds, alkyl bonds, van der Waals forces, pi-sigma, carbon, and binding residues are shown. 


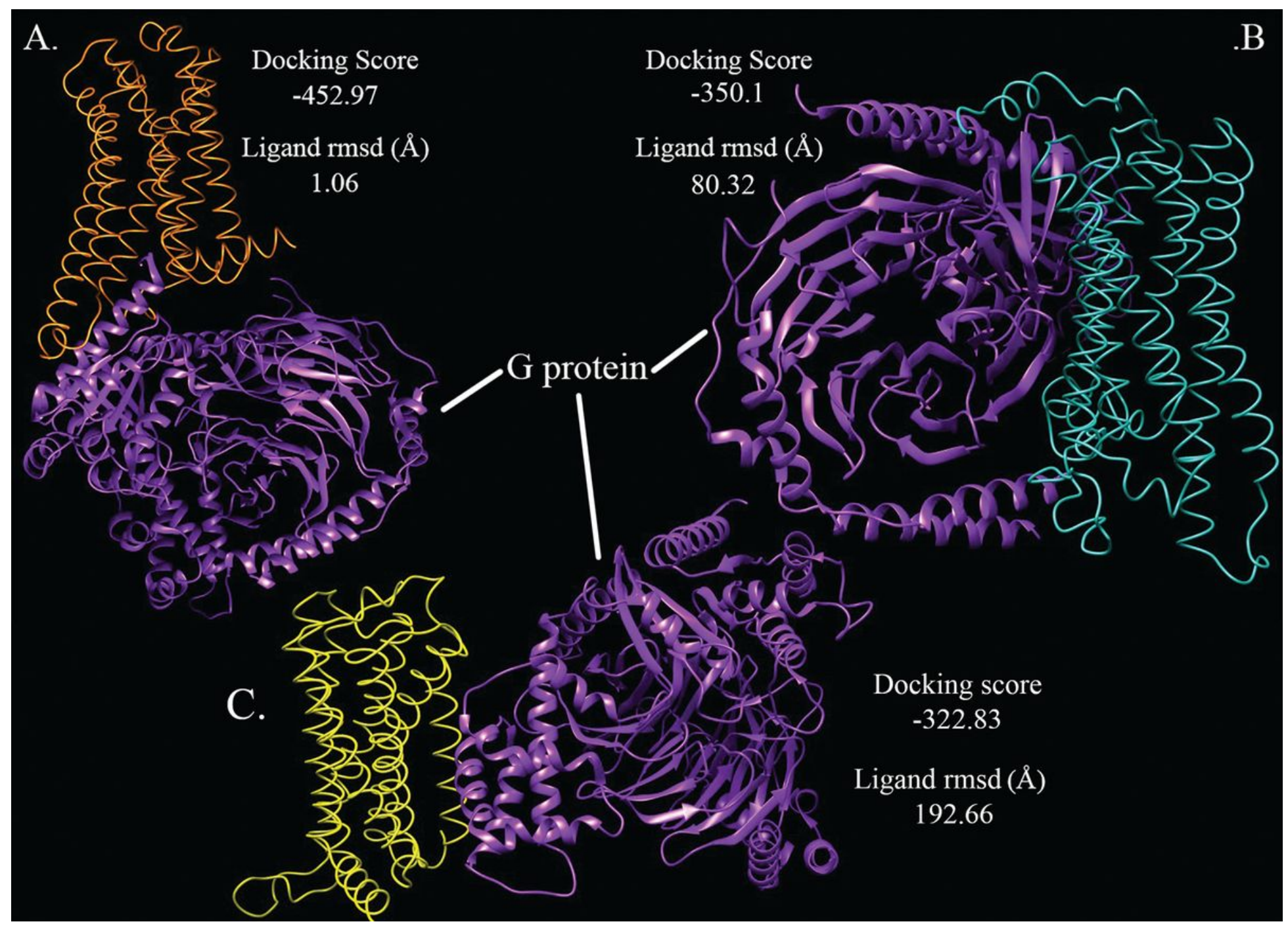

Figure 4

The HDOCK results and the binding position of G-protein and A) wild type CB2, B) I-TASSER built mutant CB2 model, and C) Phyre2 built mutant CB2 model.

\section{Supplementary Files}

This is a list of supplementary files associated with this preprint. Click to download.

- GraphicalAbstract.pdf

- Additionalfile1.docx 